\title{
Comparación de tres salinidades para evaluar el crecimiento poblacional de la microalga Chaetoceros muelleri
}

\author{
Edgar Osiris Carranza Espinal, \\ Ricardo Gómez Portillo \\ y Marvin Antonio Martínez ${ }^{1}$
}

\section{RESUMEN}

En la región sur de Honduras existen ocho laboratorios que producen 6,400 millones de poslarva de camarón por año, para lo cual usan microalgas como alimento vivo en su crianza. Por el potencial de crecimiento y valor nutricional, la especie de preferencia es la Chaetocera muelleri.

Sin embargo, los laboratorios manejan sus propios protocolos de cultivo masivo de microalgas adaptados de otros países y desconocen el efecto de la salinidad para optimizar el crecimiento de la Chaetoceros muelleri. En tal sentido, el objetivo del estudio fue comparar tres concentraciones de sal para conocer el efecto de la salinidad sobre el crecimiento de la microalga y encontrar un modelo estadístico que ayude a predecir poblaciones de microalgas.

El estudio se realizó en un laboratorio de producción de poslarva de camarón en la comunidad de Cedeño, Choluteca. Se usaron tres concentraciones de salinidad (7000, 20000 y 34000 ppm) como medio de cultivo a una densidad de $470000 \mathrm{cel} / \mathrm{cm} 3$ de inóculo y cosechando al cuarto día después de la siembra, se analizaron 108 unidades experimentales.

El mayor crecimiento de las microalgas se desarrolla a $20000 \mathrm{ppm}$ de sal a la densidad promedio de $3.85 \times 106 \mathrm{cel} / \mathrm{cm} 3$. A la salinidad de $7000 \mathrm{ppm}$, el crecimiento de las microalgas fue el menor. Es posible predecir poblaciones de microalgas usando un modelo de relación confiable ( $R 2=0.91)$, contribuyendo a optimizar los procesos de operación para los productores de poslarva de camarón.

Palabras claves: poslarva de camarón, microalgas, salinidad.

1 Profesores universitarios del Departamento de Acuicultura y Biología Marina, Centro Regional Universitario del Litoral Pacífico, UNAH: edgar.carranza@unah.edu.hn; ricardo_gomez92@yahoo.com 


\section{ABSTRACT}

In the southern region of Honduras there are eight laboratories producing 6,400 million post-larval shrimp per year, microalgae are used as live food in raising post-larval shrimp, for growth potential and nutritional value, spice preference is the Chaetocera muelleri. The laboratories manage their own protocols for mass cultivation of microalgae adapted from other countries and ignore the effect of salinity to optimize the growth of the microalgae Chaetoceros muelleri. The aim of the study was to compare three salt concentrations to determine the effect of salinity on the growth of microalgae and find a statistical model to help predict populations of microalgae. The study was conducted in a laboratory production of post-larval shrimp community Cedeño, Choluteca. Salinity three concentrations (7000, 20000 and 34000 ppm) as culture medium were used at a density of 470,000 cells / cm3 inoculum and harvesting the fourth day after seeding, 108 experimental units were analyzed. The highest growth of microalgae develops 20,000 ppm of salt to the average density of $3.85 \times 106$ cells / cm3, salinity of $7000 \mathrm{ppm}$ microalgae growth was the lowest. It is possible to predict populations of microalgae using a model of reliable relationship $(R 2=0.91)$ helping to optimize operation processes for producing post-larval shrimp.

Keywords: post-larval shrimp, algae, salinity. 


\section{INTRODUCCIÓN}

Las microalgas son usadas en acuicultura como alimento vivo para todos los estadios de crecimiento de los moluscos y crustáceos, para los peces con hábitos alimenticios omnívoros y herbívoros y en la producción de zooplancton (López, Lagunas, Jiménez y Aldaz, 2009). Los géneros de microalgas más utilizadas para acuicultura son: Isochrysis, Nannochloropsis, Chlorella, Tetraselmis, Dunaliella, Rhodomonas, Pavlova, Chaetoceros, Nitzschia y Thalassiosira (Nieves, López, Medina, Piña y Leal, 2009).

El cultivo masivo de microalgas es la fuente de alimento de mayor importancia para los organismos acuáticos cultivados comercialmente, debido a la calidad y facilidad de producir. Los géneros usados para el cultivo se han seleccionado por el potencial de crecimiento y concentración celular, tamaño, digestibilidad y valor nutricional (Alzivar y Zambrano, 2011). La composición bioquímica de las microalgas dependen de la respuesta metabólica y fisiológica de cada especie a las condiciones ambientales en donde se desarrolla su cultivo (Nieves, López, Medina, Piña y Leal, 2009). En Honduras, la especie de preferencia por los productores de poslarva de camarón es la Chaetocera muelleri.

Para estimular el crecimiento de las microalgas de agua marina comúnmente se usa el medio $f$ de Guillar y Ryther, por ser un medio económico que produce grandes cantidades de microalga (Guillard y Ryther, 1965). Pero, también para estimular el crecimiento se necesitan factores físicos y químicos como la luz, temperatura, salinidad, pH, CO2 y fotoperiodo (Lemus y otros, 2006).

Al inducir el crecimiento las microalgas se han detectado cinco etapas: la fase de ajuste, que consiste en la adaptación de las algas a las nuevas condiciones de cultivo, en esta etapa no hay crecimiento. La segunda fase es la exponencial, aquí las algas se duplican sucesivamente en intervalos iguales de tiempo (Romo, 2002). En la tercera fase llamada de retardo o declinación, las poblaciones de algas dejan de crecer por la reducción de nutrientes en el medio de cultivo, hay reducción fotosintética y poca capacidad para aprovechar la luz. La cuarta fase se conoce como estacionaria, es corta y no hay un crecimiento de las poblaciones de algas; la tasa de crecimiento es igual a la tasa de mortalidad. La quinta fase es la muerte, superando la tasa de mortalidad a la fase de crecimiento (Alvarez, 2008). La cosecha de las microalgas en los cultivos masivos es realizada al final de la fase exponencial, lo cual contando desde la etapa de inducción hasta la etapa de muerte, lleva 96 horas. 
La preferencia de los productores de cultivar Chaetocera muelleri en sus unidades productivas es por el contenido nutricional, sobre todo de ácidos grasos polinsaturados de gran beneficio para el crecimiento de la poslarva y por la facilidad de manejo del cultivo (Gutierrez, 2002). Para producir poslarvas de camarón, los acuicultores usan una combinación de alimento vivo con dietas inertes en los estadios de nauplio y zoea; los camarones son fitoplactívoros, alimentándose únicamente de microalgas cultivadas en los estadios de mysis a poslarva. La alimentación es modificada de herbívoro a omnívoro incorporando rotíferos y artemia en la dieta como alimento vivo (Pacheco, Cadena, Pilar y Tovar, 2010).

La artemia (Artemia franciscana) es cultivada para ser ofrecida como alimento a las poslarvas de camarón y durante el proceso de maduración de la artemia se alimenta con la microalga viva de Chaetoceros muelleri, porque aporta energía, facilita la digestión del depredador, estimula el crecimiento y aumenta el valor alimenticio de la Artemia sp. (Gutierrez, 2002). También esta microalga tiene preferencia de uso por ser un alimento de mejor calidad, son de menor de tamaño, posee alta sobrevivencia y contiene la mayoría de los requerimientos nutricionales que la Artemia franciscana necesita (Medina y Cordero, 1998). La Chaetoceros muelleri influye directamente en el desarrollo del sistema nervioso de las poslarvas de camarón y son precursores de muchos compuestos biológicos como las prostaglandinas que intervienen en la regulación del crecimiento y reproducción (Godínez, Hernández, Orozco y Godínez, 2003).

En la región sur de Honduras existen ocho laboratorios que producen 6,400 millones de poslarva por año, en cada laboratorio los productores manejan sus propios protocolos de cultivo masivo de microalgas que se han adaptado de otros países que tienen diferentes condiciones a las naturales del golfo de Fonseca (Andah, 2012). Muchos productores desconocen el efecto de la salinidad para optimizar el crecimiento de la microalga Chaetoceros muelleri en cuanto a horas de cultivo y densidad poblacional (células por mililitro de agua), siendo muy usual el cultivo masivo de microalgas con la salinidad del mar.

\section{OBJETIVOS}

1. Objetivo general

Conocer el efecto de la salinidad sobre el crecimiento de la microalga Chaetoceros muelleri mediante la producción masiva de microalgas para 
encontrar condiciones adecuadas de cultivo que optimicen la producción en un laboratorio de poslarva en la zona sur de Honduras.

2. Objetivos específicos

a. Observar el efecto de tres concentraciones de salinidades sobre el crecimiento poblacional de la Chaetoceros muelleri a los 1, 2, 3 y 4 días de cultivo.

b. Predecir la densidad poblacional a las 24,48 y 72 horas de cultivo para optimizar la producción de microalgas.

\section{MÉTODOS}

\section{Entorno}

El estudio se realizó en el laboratorio de producción de poslarva de camarón de BIOMARSUR, ubicado en la aldea de Cedeño, a $32 \mathrm{~km}$ al suroeste de la ciudad de Choluteca. Se usaron cepas de microalgas aisladas de Chaetoceros muelleri procedentes de un laboratorio certificado de los Estados Unidos. El experimento se manejó en tres corridas, en cada una se inoculó la microalga en nueve botellones (tres botellones para cada tratamiento de salinidad). Las microalgas se sembraron y se cosecharon en la fase exponencial del cultivo.

Una corrida es el proceso que se inicia con la siembra del inóculo de la microalga hasta la cosecha, comenzando en el día uno y cosechándose al cuarto día. Entre corridas se manejó un día de holgura para lavar y desinfectar el equipo. En total, para todo el experimento, se inocularon 27 botellones.

\section{Intervenciones}

Se estudió el efecto de las salinidades de 7 000, 20000 y 34000 partes por millón (ppm) en el crecimiento de las microalgas Chaetoceros muelleri. Para cada tratamiento se usaron tres réplicas que eran botellones cristalinos, de plástico con capacidad de 18 litros y con boca angosta, usados en la industria de agua embotellada y adaptados para el cultivo masivo de microalgas en laboratorios acuícolas.

Los botellones, antes de ser sembrados con las microalgas, se lavaron con jabón 
neutro, enjuagados con agua dulce, desinfectados con una solución de ácido clorhídrico al $10 \%$ y lavados con abundante agua salada tratada por el laboratorio de larva. El agua salada tratada consiste en agua marina filtrada en filtros de arena y carbón activado y esterilizada con luz ultravioleta. Los laboratorios de producción de poslarva manejan un alto nivel de asepsia e higiene en todas sus operaciones y el tratamiento del agua marina es un punto crítico de control en sus sistemas de calidad (FAO, 2004).

Los botellones con 7000 y 20000 ppm de sal, se llenaron de un tanque reservorio de 50 litros de agua marina filtrada a 34000 ppm de sal; la salinidad se ajustó con agua dulce para llegar a la concentración de sal deseada. El agua dulce es tratada igual que el agua marina y no lleva adición de cloro. Los botellones con 34000 ppm se llenaron directamente de los tanques reservorios de agua marina que utiliza el laboratorio larvario para sus procesos de productivos.

En la siembra se usaron tres litros de inóculo y 13 litros de agua con las salinidades establecidas, llenando los botellones hasta tener un volumen de 16 litros. La densidad del inóculo de las microalgas fue de 2.5×106 células por ml, que al añadir los 13 litros de agua se diluyeron en $\mathbf{4 7 0} 000$ células por $\mathrm{ml}$, exponiéndose a luz artificial con una intensidad luminosa de 1,800-2,000 Lux. Los cultivos se manejaron a temperatura ambiente y aireación constante (100 \% de saturación de oxígeno). Para estimular el crecimiento de las algas se fertilizó utilizando el sistema de Guillard y Ryther (1968) (ver tabla 1).

Tabla 1. Fertilizantes utilizados para la estimular la densidad poblacional de las microalgas en el cultivo masivo según Guillard y Ryther

\begin{tabular}{|lc|}
\hline Fertilizante & Partes por millón \\
\hline Silicato & 120.0 \\
\hline Tiamina & 8.0 \\
\hline B12 & 8.0 \\
\hline Biotina & 8.0 \\
\hline Cloruro férrico & 20.8 \\
\hline Cloruro de manganeso & 0.8 \\
\hline EDTA Disodio & 20.8 \\
\hline Zinc (ZnSO4) & 0.1 \\
\hline Nitrato de urea & 625.0 \\
\hline 18-46-0 & 42.5 \\
\hline
\end{tabular}


Para medir el pH del agua se usó un instrumento digital calibrado con disoluciones reguladoras de $\mathrm{pH}$ conocido de 4.0 y 7.0. La medición de la salinidad se realizó con un refractómetro, instrumento recomendado en acuicultura de agua marina para medir la salinidad del agua (Romo, 2002). Las mediciones de temperatura, pH, salinidad y oxígeno se realizaron dos veces por día.

Para contar las poblaciones de algas se extrajeron muestras diarias de $100 \mathrm{ml}$ de cada botellón, con pipetas graduadas, y se depositaron en tubos de ensayos, extrayendo con micropipetas de los tubos de ensayo un mililitro del cultivo para ser depositado en la cámara de neubauer o hematocitómetro. La concentración de las algas se determinó con el siguiente procedimiento:

Al depositar la muestra en el hematocitómetro, las células de las algas se distribuyen en cuatro cuadrantes que están enumerados y nombrados como $\mathrm{A}$, $B, C$ y $D$. El volumen de agua de cada cuadrante del hematocitómetro es de $1 / 10,000 \mathrm{ml}$.

Se cuentan las células de cada cuadrante, se multiplica con un factor exponencial y se divide entre cuatro, ejemplo: $\frac{A+B+C+D \times 10^{4}}{4}$

Las lecturas de las algas es en células/mililitro (cel/ml).

El conteo de las algas se logró con un microscopio compuesto, usando un lente de $10 x$ y fijado en fresco.

\section{Diseño}

El diseño de la investigación fue correlacional y experimental, se observó el crecimiento y densidad de las algas en función del tiempo de cultivo, las variables y las unidades experimentales fueron asignadas aleatoriamente, considerado un experimento verdadero.

En la evaluación experimental se compararon los tratamientos que fueron los crecimientos poblacionales diarios de la Chaetoceros muelleri sembrados en las tres concentraciones de salinidad del medio del cultivo. También se usó un modelo de regresión polinómica para predecir las poblaciones de microalgas a los 48 y 72 horas de cultivo. El modelo de regresión polinómica es el que más se ajusta por el crecimiento y desarrollo de las microalgas, la predicción de la densidad de células por mililitro de agua es importante en un aboratorio de larva, ya que se ajusta el volumen 
de alimento natural que se le da a la poslarva de camarón.

Análisis estadístico

Para encontrar el efecto de la salinidad sobre las muestras, se usó el diseño de medidas repetidas en el tiempo, evaluando tres salinidades, cuatro días de cultivo y nueve repeticiones (muestras por triplicado en tres corridas), logrando en total 108 unidades experimentales analizadas al 0.05 de nivel de significancia. Para la comparación múltiple de medias se utilizó la prueba diferencia mínima significa (DMS), detectando la mejor salinidad como medio de cultivo y el día de mayor producción de microalgas $(\alpha=0.05)$.

Para la predicción de las poblaciones de microalgas, según los días de incubación, se usó el análisis de regresión cuadrática (análisis de relación) con su respectiva ecuación de predicción. En los análisis se manejó el software estadístico de Infostat@2011.

\section{RESULTADOS}

Efecto de la salinidad sobre la densidad de algas

La salinidad y los días de cultivo mostraron resultados concluyentes $(P<0.0001)$, de igual manera la interacción de los días de cultivo y salinidad como medio de cultivo $(P<0.0001)$, es decir, las tres concentraciones de salinidad sometidas a prueba y los cuatro días de cultivo mostraron poblaciones diferentes de microalgas; la variabilidad de todos los datos encontrados en el experimento no superó el $8.7 \%$ como coeficiente de variación.

La mayor población de microalgas se logró a los cuatro días de cultivo (72 horas), incrementándose desde el día de siembra hasta el día cuatro $(P<0.05)$, que fue el día de cosecha.

La mayor densidad de microalgas se encontró a 20,000 ppm de sal en el medio de cultivo, con una densidad promedio para las tres corridas de 3, 84, 5490.67 células por mililitro (ver tabla 2). Entre corridas no se encontraron resultados concluyentes $(P>0.6653)$ mostrando el mismo patrón en cada una de ella. 
Tabla 2. Densidad poblacional en células por mililitro de la microalga Chaetoceros muelleri cultivada en un laboratorio de producción de poslarva en la zona sur de Honduras

\begin{tabular}{|rrrr} 
& \multicolumn{3}{c|}{ Salinidad en el medio de cultivo expresado en partes por millón (mg/L) } \\
\cline { 2 - 4 } Días de cultivo & 7000 & 20000 & 34000 \\
\hline 1 & $470,000 \mathrm{i}$ & $470,000 \mathrm{i}$ & $470,000 \mathrm{i}$ \\
2 & $725,742.67 \mathrm{~h}$ & $2,221,912.44 \mathrm{f}$ & $1,759,709.56 \mathrm{~g}$ \\
3 & $1,376,533.44 \mathrm{~d}$ & $3,552,288.67 \mathrm{~b}$ & $2,633,309.67 \mathrm{c}$ \\
4 & $2,433,975.11 \mathrm{e}$ & $3,845,490.67 \mathrm{a}$ & $3,559,080.33 \mathrm{~b}$ \\
\hline
\end{tabular}

Letras distintas indican diferencias significativas $(P<0.05)$.

El menor crecimiento de las microalgas se observó a la salinidad de 7000 ppm en los cuatro días de cultivo. En el cultivo masivo de Thalassiosira fluviatilis se encontró la mejor densidad celular a 35000 ppm de salinidad (Alzívar y Zambrano, 2011). Pacheco y otros (2010) descubrieron que las microalgas marinas limitan su reproducción celular a salinidades menores a las 20000 ppm.

Figura 1. Crecimiento poblacional de las microalgas Chaetoceras muelleri sembradas a 7000,20000 y 34000 ppm de sal durante cuatro días de cultivo

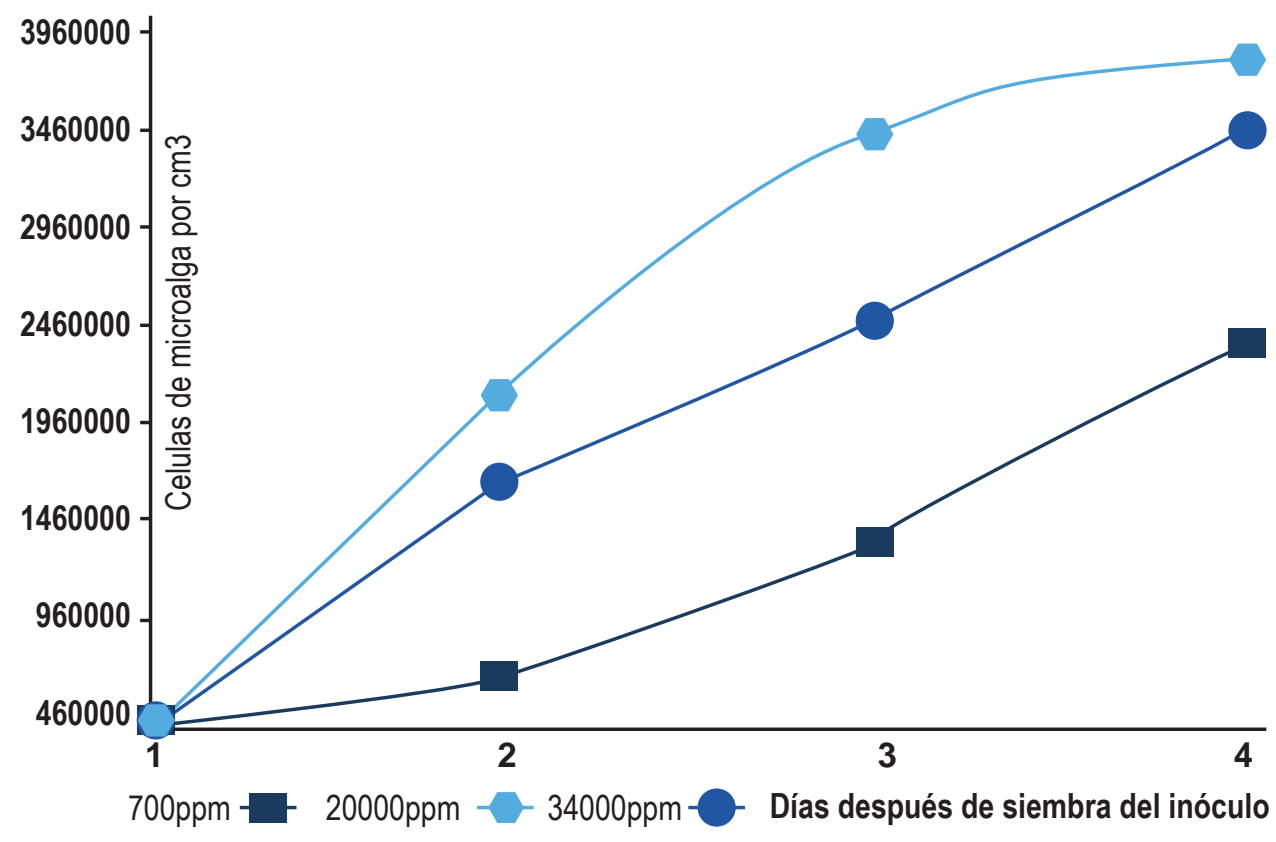


La salinidad mínima y máxima que toleran las Chaetoceros es entre 7000 y 50000 ppm (Nieves, López, Medina, Piña y Leal, 2009), a 7000 ppm se demostró en el estudio que las microalgas crecen a un ritmo menor, pero se desarrollan hasta llegar a la fase estacionaria según su ciclo de crecimiento, mostrando comportamiento parecido con los otros medios de cultivo (ver figura 1).

Estimación de la densidad de algas a las 24, 48 y 72 horas de cultivo

En las 108 muestras analizadas se encontraron diferencias significativas $(P<0.001)$ en las poblaciones de microalgas desarrolladas a las 24, 48 y 72 horas después de la siembra del inóculo en los tres tratamientos de salinidad. Al llegar al día dos después de siembra (24 horas), la densidad celular de la siembra $(470,000 \mathrm{cel} / \mathrm{cm} 3)$ en promedio se incrementó en un $19.6 \%$; a las 48 horas las poblaciones de microalgas aumentaron un $33.6 \%$ más (53.2 \%) y el mayor crecimiento poblacional se observó a las 72 horas pos siembra con el $46.7 \%$.

A las 72 horas de cultivo fue donde se encontró la mayor precisión para estimar la densidad de microalgas según la salinidad del medio y los días después de la siembra; a las 24 horas de cultivo el modelo para estimar fue el menos preciso, pero por los valores encontrados en el factor de determinación (R2) estadísticamente el modelo es aceptable para hacer estimaciones de poblaciones de microalgas (ver tabla 3)

Tabla 3. Ecuaciones de regresión polinómica para predecir poblaciones de la microalga Chaetocera muelleri, manejadas a tres tiempos de cultivo

\begin{tabular}{|llc|}
\hline Tiempo de cultivo & \multicolumn{1}{c}{ Ecuación de predicción } & R2 \\
\hline 24 horas & $\breve{y}=-738478.9+1099221.56 a+131.6 b-0.0027 b^{2}$ & 0.79 \\
48 horas & $\breve{y}=-1485704.83+1025355.30 a+221.19 b+0.0047 b^{2}$ & 0.81 \\
72 horas & $\breve{y}=-1501069.24+938013.51 a+225.30 b-0.0047 b^{2}$ & 0.91
\end{tabular}

a=salinidad manejada en el medio de cultivo; b=días después de la siembra del inóculo 


\section{DISCUSIÓN}

El mayor crecimiento de las microalgas se encontró a 20000 ppm de sal, desarrollando 1.6 veces de población de microalgas por $\mathrm{ml}$ que las encontradas en 7000 ppm. A 34000 ppm de salinidad las microalgas crecieron en promedio $8.2 \%$ menos que a 20000 ppm en el cuarto día de cultivo. Las microalgas son tolerantes a las altas concentraciones de sal, a pesar de que la tasa de reproducción disminuye al incrementar la sal en el agua (Nieves, López, Medina, Piña y Leal, 2009).

Cuando la salinidad en el medio de cultivo está por debajo de las 20000 ppm, las células de las microalgas desarrollan condiciones hipo osmóticas que reducen la tasa de crecimiento y actividad metabólica (Azay, López, Lázaro y Nodar, 2009), siendo esta la causa de que las poblaciones de microalgas a 7000 ppm de salinidad sean las más bajas del experimento.

Molina y otros (2007) encontraron que la Rhodosorus marinus inhibe su crecimiento a salinidades inferiores a las $15000 \mathrm{ppm}$ de sal y el crecimiento de la microalga es duplicado en salinidades de 16000 y 25000 ppm, logrando el óptimo desarrollo poblacional a 35000 ppm de salinidad. También se observó este comportamiento en el crecimiento de Chroomonas sp. como microalga para alimento de camarón cultivado en Venezuela, en salinidades de 20000 y 35000 ppm, obteniendo el mayor crecimiento celular en clorofila y caratenoides (Bermudez, Lodeiros y Morales, 2002).

En las estimaciones de poblaciones de microalgas por $\mathrm{cm} 3$, para los productores es muy importante conocer las poblaciones que podrían cosechar en la fase estacionaria del cultivo de la Chaetocera muelleri, puesto que muchos laboratorios alimentan diariamente a las poslarvas de camarón con las microalgas y para hacerlo necesitan de logística y coordinación para realizar la actividad. Las predicciones de las poblaciones de microalgas a las 48 y 72 horas de cultivo hacen del sistema productivo más eficiente, optimizando recursos como tiempo, energía y talento humano, que contribuyen a reducir costos de producción para el productor. 


\section{CONCLUSIONES}

La salinidad tiene un efecto directo en el crecimiento poblacional de la Chaetocera muelleri, los mayores crecimientos se observaron en 20000 ppm de sal con 3.85x106 $\mathrm{cel} / \mathrm{cm} 3$ en el cuarto día de cultivo. Las microalgas desarrollan condiciones hipo osmóticas que reducen la actividad celular a salinidades de 7000 ppm, encontrando una población de $2.43 \times 106 \mathrm{cel} / \mathrm{cm} 3$.

La mayor precisión en la estimación de poblaciones de microalgas se encontró al cuarto día de cultivo (72 horas) con un R2=0.91 ( $=-1501069.24+938013.51 \mathrm{a}+225.30 \mathrm{~b}$ -0.0047b2), a los dos días de cultivo la densidad celular se incrementa en un $19.6 \%$; al tercer día en $53.2 \%$ y en el cuarto día alcanzan el $100 \%$ del crecimiento poblacional estimado. Por lo que es posible hacer predicciones de cosecha de microalgas a partir de la concentración de salinidad que se usa en el medio y los días después de la siembra del inóculo.

\section{BIBLIOGRAFÍA}

Álvarez, H. G. (2008). Introducción al método ficológico. Ecuador: Escuela Superior Politecnica del Litoral. Obtenido de http://mail- cenaim.espol.edu.ec/publicaciones/algas/capitulo_3.pdf

Alzivar, E., \& Zambrano, J. Á. (2011). Utilización de la microalga marina Thalasiosira pseudomona como alternativa de alimentación y sobrevivencia de camarones cultivados en cautiverio en mata, Ecuador. Manta, Ecuador: Universidad Laica Eloy Alfaro de Manabí.

Andah. (2012). El desarrollo de la larvicultura de 1990 al 2012 en la zona dur de Honduras. Asociación Nacional de Acuícultores de Honduras. Choluteca.

Azay, M. G., López, T. R., Lázaro, A. V., \& Nodar, R. P. (abril de 2009). Evaluation of the growth of Thalassiosira fluviatilis in three different salinities. REDVET, 10(4), 4-12. Obtenido de http://www.veterinaria.org/revistas/redvet/n040409/040918

Bermúdez, J. L., Lodeiros, C., \& Morales, E. (2002). Producción de biomasa de la microalga marina Chroomonas sp., en funcón del $\mathrm{pH}$, intensidad luminosa y salinidad. Invemar(31), 167-185. Recuperado el 05 de diciembre de 2014

FAO. (2004). Manejo sanitario y mantenimiento de la bioseguridad de los laboratorios de postlarvas de camarón blanco (Penaeus vannamei) en América Latina 
( $1^{\circ}$ ed.). (D. d. Pesqueros, Ed.) Roma, Italia: Organización de las Naciones Unidas para la Agricultura y la Alimentación. Recuperado el Junio de 2014, de www.fao.org

Godínez, D., Hernández, A., Orozco, J., \& Godínez, E. (abril de 2003). Valoración entre la tasa de ingestión y supervivencias de larcas de camaron azul nutridas con diferentes concentraciones de Chaetoceros calcitrans. Zootecnia tropical, 21(2), 37-48. Obtenido de http://www.scielo.org.ve/scielo.phd=S0798-7269200 30002000 03\&script=sci_arttext

Guillard, R. y Ryther, J. (1965). Culture of Phytoplankton for Feeding Marine Invertebrates. Recuperado el 04 de abril de 2014, de http://mel.xmu.edu.cn/UserFiles /Image/gomap/pdf/f_2_medium.pdf

Gutiérrez, I. P. (2002). Estimación de los requerimientos alimenticios para el crecimiento de briapódo Artmia franciscana alimentado con la diatomea Chaoteceros muelleri orientada a la producción masica. Manzanillo, Colima, Méixco: Universidad de Colima.

Lemus, N., Urbano, T., Arredondo-Vega, B., Guevara, M., Vásque, A., L. C.-P., \& Vallejo, N. (septiembre de 2006). Crecimiento y perfil bioquímico de Chaetoceros muelleri cultivadas en sistemas continuos y semidiscontinuos. Ciencias Marinas, 32(3), 597-603. Obtenido de http://www.redalyc.org/pdf/480/48032311.pdf

López, J. A., Lagunas, N. G., Jiménez, L. R., \& Aldaz, N. H. (2009). Crecimiento de la diatomea Thalassiosira pseudonana en cultivos estáticos con iluminaión continua y fotoperiodo a diferentes salinidades. Biotecnia, XI(1), 18. Recuperado el 20 de noviembre de 2014

Medina, C. E., \& Cordero, B. (septiembre de 1998). Crecimiento y composición bioquímica de la diatomea Chaetoceros muelleri Lemmerman, mantenida en cultivo estático en un medio comercial. Ciencia y mar, 2(6), 19-25. Obtenido de http://www.umar.mx/revistas/6/chaetoceros.pdf

Molina, L., Jonte, L., Mora, R., Ortega, J., \& Morales, E. (30 de abril de 2007). Salinity influence on growth of the Rhodosorus marinus (Rhodophyta)microalgae in batch cultures. LUZ(2007 (24)), 249-253. Recuperado el septiembre de 2014

Nieves, M., López, D., Medina, A., Piña, P., \& Leal, S. (2009). Producción y calidad de Chaetoceros muelleri a diferentes concentraciones de nutrientes y densidad de inóculos. Investigación Marina, 30(2), 123-133. Obtenido de http://www.cim.uh.cu/rim/pdf/2009/2/Medio\%20f.pdf

Pacheco, J. M., Cadena, M. A., Pilar, M. d., \& Tovar, D. (2010). Effect of culture medium and nutrient concentration on fatty acid content of Chaetoceros muelleri. (E. Olguín, Ed.) Revista Latinoamericana de Biotecnología Ambiental y Algal, 1(1), 6-10. Obtenido de http://www3.inecol.edu.mx/solabiaa/ARCHIVOS/documentos/reportes/RELBAA_-_V1N1.pdf 
Romo, A. K. (2002). Manual para el cultivo de microalgas. Biología Marina. La Paza, Baja California, México: Universidad Autónoma de Baja California Sur. Recuperado el 22 de febrero de 2014, de http://biblio.uabcs.mx/tesis/te1366.pdf 